\title{
L1521E: The first starless core with no molecular depletion
}

\author{
M. Tafalla and J. Santiago \\ Observatorio Astronómico Nacional, Alfonso XII 3, 28014 Madrid, Spain \\ Received 20 November 2003 / Accepted 22 December 2003

\begin{abstract}
L1521E seems unique among starless cores. It stands out in a distribution of a ratio $(R)$ that we define to asses core evolution, and which compares the emission of the easily-depleted $\mathrm{C}^{18} \mathrm{O}$ molecule with that of the hard to deplete, late-time species $\mathrm{N}_{2} \mathrm{H}^{+}$. While all cores we have studied so far have $R$ ratio lower than 1, L1521E has an $R$ value of 3.4, which is 8 times the mean of the other cores. To understand this difference, we have modeled the $\mathrm{C}^{18} \mathrm{O}$ and $\mathrm{N}_{2} \mathrm{H}^{+}$abundance profiles in L1521E using a density distribution derived from $1.2 \mathrm{~mm}$ continuum data. Our model shows that the $\mathrm{C}^{18} \mathrm{O}$ emission in this core is consistent with constant abundance, and this makes L1521E the first core with no $\mathrm{C}^{18} \mathrm{O}$ depletion. Our model also derives an unusually low $\mathrm{N}_{2} \mathrm{H}^{+}$abundance. These two chemical peculiarities suggest that $\mathrm{L} 1521 \mathrm{E}$ has contracted to its present density very recently, and it is therefore an extremely young starless core. Comparing our derived abundances with a chemical model, we estimate a tentative age of $\leq 1.5 \times 10^{5} \mathrm{yr}$, which is too short for ambipolar diffusion models.
\end{abstract}

Key words. ISM: abundances - ISM: clouds - ISM: molecules - stars: formation - ISM: individual: L1521E

\section{Introduction}

Observations of starless dense cores over the last few years have shown that molecular depletion onto cold dust grains is a common feature in low-mass star-forming regions (e.g., Caselli et al. 1999; Tafalla et al. 2002). As a core contracts, the amount of depletion of certain molecules like CS and CO increases with time (e.g., Bergin \& Langer 1997; Aikawa et al. 2003), so we can expect that depletion will provide an accurate clock for core evolution when its details are well understood. Even before this is a reality, depletion can be used as a qualitative time marker, in the sense that evolved cores should be more depleted of certain species than younger cores. When we attempt to build a sequence of cores having different amounts of depletion, however, we find that the population of undepleted (or lightly) depleted cores is missing. These undepleted cores should represent the earliest stages of core contraction, and probably they have not been identified so far because of an observational bias. Given the interest of young cores for studies of core contraction, finding a member of this population is an urgent challenge.

To identify cores with low degree of depletion, we have started a systematic study of cores with weak $\mathrm{NH}_{3}$ lines in the survey of Benson \& Myers (1989), as previous observations of cores bright in $\mathrm{NH}_{3}$ have only found cases of strong depletion (e.g., Caselli et al. 1999; Tafalla et al. 2002). Among our targets, the L1521E core in Taurus has revealed itself as a core with negligible depletion, and here we report a preliminary analysis of its properties. This core has already been identified as a very young core by Hirota et al. (2002), who found

Send offprint requests to: M. Tafalla, e-mail: m.tafalla@oan.es it very prominent in carbon-chain molecules, comparable to TMC-1 (see also Aikawa et al. 2003). In this paper we show that L1521E has the lowest measured level of $\mathrm{C}^{18} \mathrm{O}$ depletion, and in a forthcoming article we will present the results of an ongoing chemical survey of this core.

\section{Observations}

We observed L1521E in $\mathrm{C}^{18} \mathrm{O}(1-0)$ and $\mathrm{N}_{2} \mathrm{H}^{+}(1-0)$ with the FCRAO $14 \mathrm{~m}$ telescope during 2002 March. The observations were done with the SEQUOIA array in on-the-fly position switching mode. The facility correlator provided a velocity resolution of about $0.07 \mathrm{~km} \mathrm{~s}^{-1}$, and all data were converted into the main beam brightness scale assuming an efficiency of 0.55 . The telescope beam size was approximately $50^{\prime \prime}$.

We observed L1521E with the IRAM $30 \mathrm{~m}$ telescope in 2003 September (lines) and October (continuum). The line observations consisted of maps in $\mathrm{N}_{2} \mathrm{H}^{+}(1-0)$ and $\mathrm{C}^{18} \mathrm{O}(2-1)$, and crosses in $\mathrm{C}^{17} \mathrm{O}(1-0), \mathrm{C}^{17} \mathrm{O}(2-1)$, and $\mathrm{C}^{34} \mathrm{~S}(2-1)$. All data were taken in frequency switching mode with a velocity resolution of $0.03 \mathrm{~km} \mathrm{~s}^{-1}$, and were converted into main beam brightness units using standard efficiencies. The $1.2 \mathrm{~mm}$ continuum observations were done with MAMBO-2 in on the fly mode. Sky dips were used to measure the atmospheric extinction, and observations of planets were used for calibration. The resolution of the $30 \mathrm{~m}$ telescope varies from $26^{\prime \prime}$ for $\mathrm{N}_{2} \mathrm{H}^{+}(1-0)$ to $11^{\prime \prime}$ for $\mathrm{C}^{18} \mathrm{O}(2-1)$ and the $1.2 \mathrm{~mm}$ continuum.

\section{The $R$ ratio and the uniqueness of L1521E}

To search for cores at the earliest stages of contraction, it is convenient to define a simple observable that measures 

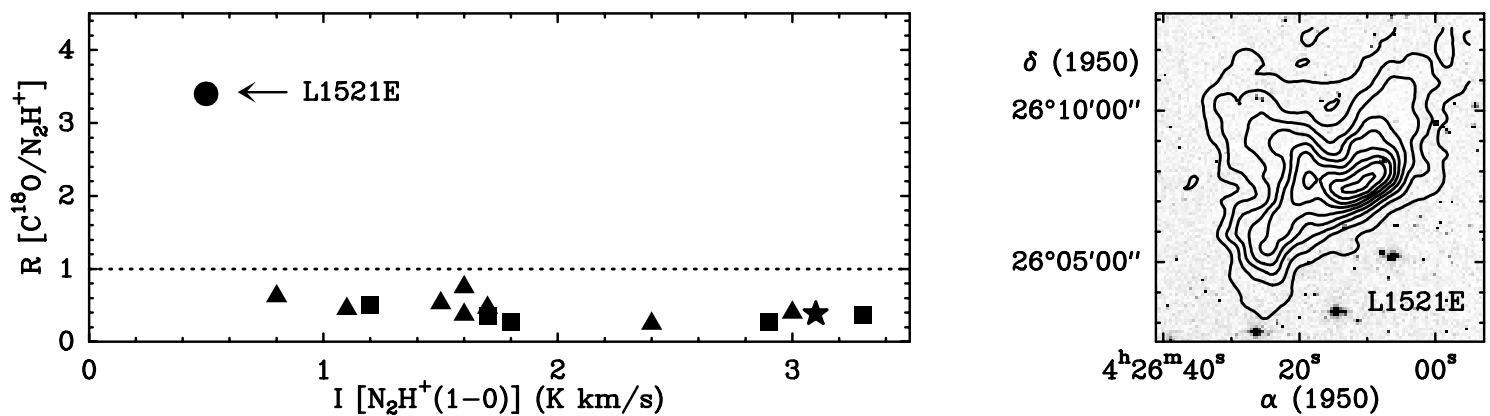

Fig. 1. Left: $R$ parameter $\left(\mathrm{C}^{18} \mathrm{O}(1-0) / \mathrm{N}_{2} \mathrm{H}^{+}(1-0)\right.$ ratio at the core center) as a function of the central $\mathrm{N}_{2} \mathrm{H}^{+}(1-0)$ integrated intensity. Each point represents a core and the dashed line at $R=1$ represents the average $R$ value for the core models with no $\mathrm{C}^{18} \mathrm{O}$ depletion in Tafalla et al. (2002). The squares represent cores studied in that paper (L1400K, L1517B, L1498, L1495, and L1544 by increasing $\mathrm{N}_{2} \mathrm{H}^{+}$intensity), the triangles represent cores from an unpublished survey (L981-1, L1197, L1155C1S, L1155C1N, L1512, L234E-1, L1333, and L694-2, same order), and the star symbol indicates B335, the only core with a YSO. Note how L1521E stands out from the core sample. Right: large scale $\mathrm{C}^{18} \mathrm{O}(1-0)$ integrated intensity map overlayed on a DSS blue image. Contours are $.2, .4, \ldots \mathrm{K} \mathrm{km} \mathrm{s}^{-1}$. All radio data are from FCRAO.

depletion without the need of a complicated radiative transfer calculation. One possibility is to compare the emission of two molecules known to have different depletion behaviors at typical core densities, and for this we choose $\mathrm{C}^{18} \mathrm{O}$ and $\mathrm{N}_{2} \mathrm{H}^{+}$. $\mathrm{C}^{18} \mathrm{O}$ is a heavy depleter at densities of a few $10^{4} \mathrm{~cm}^{-3}$, while $\mathrm{N}_{2} \mathrm{H}^{+}$seems to remain in the gas phase up to densities of about $10^{6} \mathrm{~cm}^{-3}$ (e.g., Caselli et al. 1999; Tafalla et al. 2002). Thus, if we define $R\left[\mathrm{C}^{18} \mathrm{O} / \mathrm{N}_{2} \mathrm{H}^{+}\right]$( $R$ hereafter) as the ratio between the integrated intensities of $\mathrm{C}^{18} \mathrm{O}$ and $\mathrm{N}_{2} \mathrm{H}^{+}$at the center of a core, we expect $R$ to be a sensitive indicator of depletion. In particular, we expect $R$ to be low in cores with strong $\mathrm{C}^{18} \mathrm{O}$ depletion and high in cores with little $\mathrm{C}^{18} \mathrm{O}$ depletion.

Our definition of $R$ has the additional advantage that it compares two molecules with different gas-phase formation time scales: $\mathrm{C}^{18} \mathrm{O}$ forms relatively quickly in the core history while $\mathrm{N}_{2} \mathrm{H}^{+}$is a late-time molecule (e.g., Aikawa et al. 2003). Thus, if chemical youth is correlated with lack of depletion, the $R$ ratio should be further enhanced in young cores because of their relatively lower $\mathrm{N}_{2} \mathrm{H}^{+}$abundances. A signature of youth and lack of depletion, therefore, is the combination of a large $R$ and a relatively low $\mathrm{N}_{2} \mathrm{H}^{+}$intensity.

To calibrate the $R$ ratio, we test its behavior in a sample of cores selected for their strong $\mathrm{N}_{2} \mathrm{H}^{+}$or $\mathrm{NH}_{3}$ emission, and therefore likely to be evolved. This sample includes the 5 cores studied in Tafalla et al. (2002), a set of 8 additional cores from an unpublished survey, and a core with an embedded YSO, B335. All these cores have been mapped in $\mathrm{C}^{18} \mathrm{O}$ and $\mathrm{N}_{2} \mathrm{H}^{+}$with the FCRAO telescope, and their $R$ ratio has been estimated at the position of maximum $\mathrm{N}_{2} \mathrm{H}^{+}$emission. In addition and as a reference, we have estimated the $R$ ratio for the 5 Monte Carlo core models with constant $\mathrm{C}^{18} \mathrm{O}$ abundance in Tafalla et al. (2002), finding an average $R$ value of 1.0.

As Fig. 1 illustrates, all cores in our sample lie below the $R=1$ line despite their factor of 4 spread in $\mathrm{N}_{2} \mathrm{H}^{+}(1-0)$ integrated intensity. The sample has a mean $R$ value of 0.43 with an rms of 0.14 , and its location under the $R=1$ line is suggestive of strong $\mathrm{C}^{18} \mathrm{O}$ depletion (a conclusion supported by detailed radiative transfer modeling of some objects). This systematic behavior suggests that $R=1$ can be taken as a reasonable boundary between cores with and without $\mathrm{C}^{18} \mathrm{O}$ depletion, and that a search for undepleted cores should look for objects above the $R=1$ line. Thus, over the last few years we have carried out a search for high- $R$ cores using the FCRAO telescope, mapping core candidates both in $\mathrm{N}_{2} \mathrm{H}^{+}(1-0)$ and $\mathrm{C}^{18} \mathrm{O}(1-0)$. Although the search is still ongoing and several candidates seem promising, one object stands out because it combines the highest $R$ value and the lowest $\mathrm{N}_{2} \mathrm{H}^{+}$intensity. This core, L1521E, shown in Fig. 1 as the outlying point labeled with its name, has $R=3.4$, which is 8 times larger than the mean or 20 times the rms. These characteristics make L1521E the best candidate we know of to an undepleted core, and as we will in the following section, a detailed analysis of its molecular composition confirms this interpretation.

\section{The internal structure of L1521E}

The high $R$ ratio of L1521E can arise from a high central $\mathrm{C}^{18} \mathrm{O}$ abundance, a low $\mathrm{N}_{2} \mathrm{H}^{+}$fraction, a combination of both, or some excitation effect (after all, the two molecules have very different dipole moments). To discern between these options, we now solve the radiative transfer of the two species and determine their abundance profiles. For this, we rely on observations of the $1.2 \mathrm{~mm}$ continuum, $\mathrm{C}^{18} \mathrm{O}(1-0), \mathrm{C}^{18} \mathrm{O}(2-1), \mathrm{C}^{17} \mathrm{O}(1-0)$, $\mathrm{C}^{17} \mathrm{O}(2-1)$, and $\mathrm{N}_{2} \mathrm{H}^{+}(1-0)$, shown in part in Fig. 2 .

\subsection{Physical parameters}

We first derive the density profile of L1521E from its distribution of the $1.2 \mathrm{~mm}$ continuum emission. This emission is optically thin and appears unaffected by chemical differentiation or excitation effects, so it can be easily inverted into a density distribution (e.g., André et al. 1996). As Fig. 2 shows, the $1.2 \mathrm{~mm}$ emission (like that of the molecules) is elongated NW-SE, and therefore deviates from spherical symmetry. Thus, to derive a density distribution that can be used by our sphericallysymmetric Monte Carlo radiative transfer analysis (see below), we first average elliptically the observed emission, assuming an aspect ratio of $2: 1$ and a position angle of $-60^{\circ}$. Then, we find an analytic density profile that after simulating an on-thefly observation predicts the correct radial profile of $1.2 \mathrm{~mm}$ 


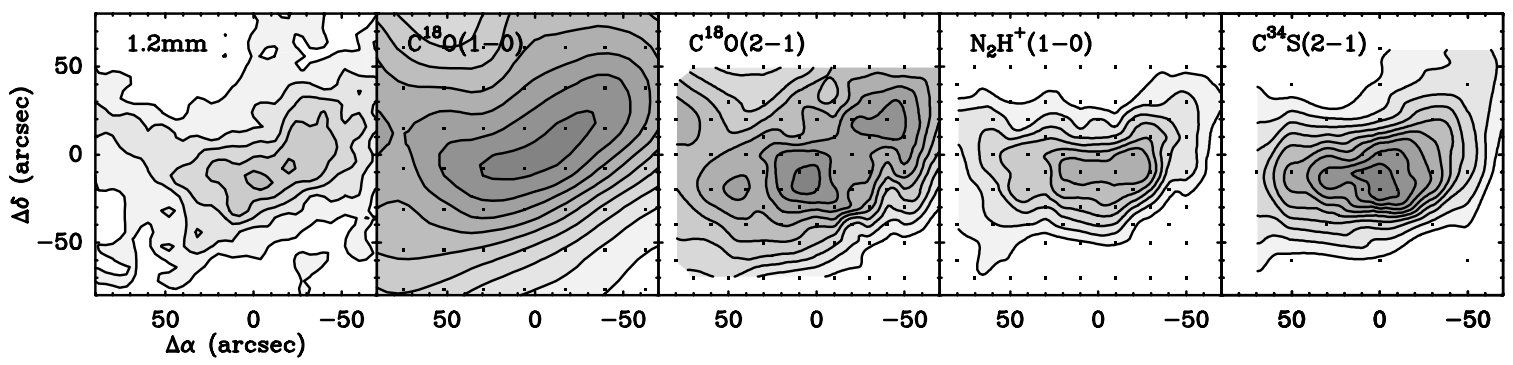

Fig. 2. Maps of L1521E in $1.2 \mathrm{~mm}$ continuum, $\mathrm{C}^{18} \mathrm{O}(1-0), \mathrm{C}^{18} \mathrm{O}(2-1), \mathrm{N}_{2} \mathrm{H}^{+}(1-0)$, and $\mathrm{C}^{34} \mathrm{~S}(2-1)$. Note the similar distribution of all tracers, something not seen in cores with depletion. First contour and contour spacing are $4 \mathrm{mJy} \mathrm{beam}^{-1}$ for the $1.2 \mathrm{~mm}$ continuum map, $0.2,0.3,0.1$, and $0.1 \mathrm{~K} \mathrm{~km} \mathrm{~s}^{-1}$ for $\mathrm{C}^{18} \mathrm{O}(1-0), \mathrm{C}^{18} \mathrm{O}(2-1), \mathrm{N}_{2} \mathrm{H}^{+}(1-0)$, and $\mathrm{C}^{34} \mathrm{~S}(2-1)$, respectively. Offsets are referred to $\alpha_{2000}=4^{\mathrm{h}} 29^{\mathrm{m}} 15^{\mathrm{s}} .7, \delta_{2000}=26^{\circ} 14^{\prime} 5^{\prime \prime}$. Note that the $\mathrm{C}^{34} \mathrm{~S}(2-1)$ map is heavily undersampled. The $\mathrm{C}^{18} \mathrm{O}(1-0)$ map is from FCRAO data and the other maps are from IRAM $30 \mathrm{~m}$ data.
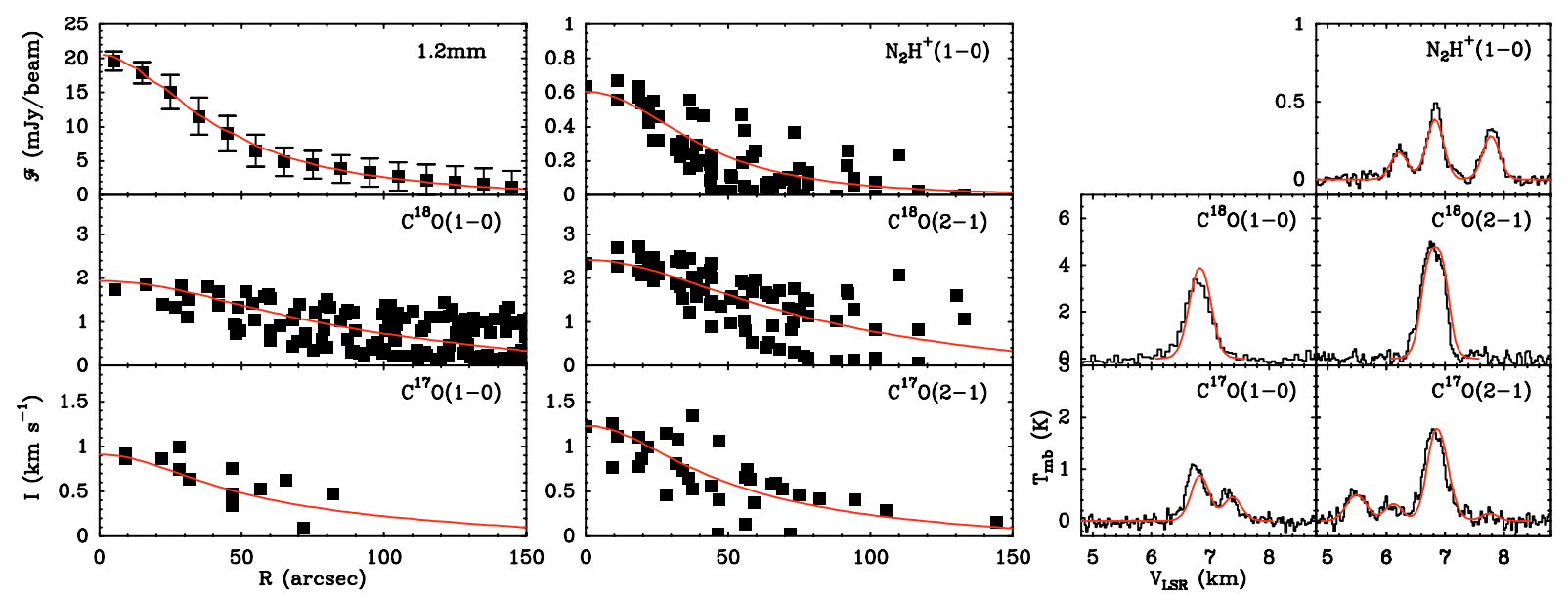

Fig. 3. Radial profiles of integrated intensity (left) and central emerging spectra (right) for $1.2 \mathrm{~mm}$ continuum, $\mathrm{N}_{2} \mathrm{H}^{+}(1-0)$, $\mathrm{C}^{18} \mathrm{O}(1-0)$, $\mathrm{C}^{18} \mathrm{O}(2-1), \mathrm{C}^{17} \mathrm{O}(1-0)$, and $\mathrm{C}^{17} \mathrm{O}(2-1)$ (data origin as in Fig. 2). The squares and histograms are observed data, and the solid lines are model predictions. The model fitting the $1.2 \mathrm{~mm}$ continuum is the basis of our core density profile, and the models fitting the line emission have been calculated using a Monte Carlo radiative transfer model. All models assume constant molecular abundance (see text).

continuum emission (see Tafalla et al. 2002 for details). For consistency with previous work, we assume a dust emissivity $\kappa_{1.2 \mathrm{~mm}}=0.005 \mathrm{~cm}^{2} \mathrm{~g}^{-1}$ and a dust temperature of $10 \mathrm{~K}$ (see below). In this way, we derive a density distribution of the form $n(r)=n_{0} /\left(1+\left(r / r_{0}\right)^{\alpha}\right)$, where $n_{0}=2.7 \times 10^{5} \mathrm{~cm}^{-3}$, $r_{0}=6.3 \times 10^{16} \mathrm{~cm}\left(=30^{\prime \prime}\right.$ at $\left.140 \mathrm{pc}\right)$, and $\alpha=2$. Figure 3 shows that this profile provides a reasonable fit to the observed $1.2 \mathrm{~mm}$ continuum emission.

The $\mathrm{NH}_{3}$ emission from L1521E is extremely weak (Benson \& Myers 1989; Hirota et al. 2002), so we rely on $\mathrm{C}^{17} \mathrm{O}$ to determine the gas kinetic temperature. $\mathrm{C}^{17} \mathrm{O}$ seems undepleted at the core center (see below), and fits to its hyperfine structure indicate that the emission is optically thin. Thus, we can convert the observed central 2-1/1-0 intensity ratio $(\approx 1.3)$ into an excitation temperature $(\approx 9 \mathrm{~K})$ which we approximate as $10 \mathrm{~K}$. The $2-1 / 1-0$ line ratio seems constant with radius, so the excitation temperature must also be close to constant. Given the low dipole moment of $\mathrm{C}^{17} \mathrm{O}(0.1 \mathrm{D})$, this excitation temperature should be a good approximation to the gas kinetic temperature, and we will assume in our modeling a constant gas temperature of $10 \mathrm{~K}$.

Finally, we characterize the velocity field of L1521E using hyperfine fits to its $\mathrm{N}_{2} \mathrm{H}^{+}(1-0)$ emission. These fits indicate an intrinsic $\mathrm{N}_{2} \mathrm{H}^{+}$linewidth of $0.3 \mathrm{~km} \mathrm{~s}^{-1}$, which means a nonthermal component of about $0.27 \mathrm{~km} \mathrm{~s}^{-1}$ (FWHM). When modeling the $\mathrm{C}^{18} \mathrm{O}(1-0)$ lines, a slightly larger linewidth is needed in the outer core, and we have parametrized this behavior as a linear increase in the non thermal component up to $0.58 \mathrm{~km} \mathrm{~s}^{-1}$ at $4 \times 10^{17} \mathrm{~cm}$ (the core outer radius in our model).

\subsection{Molecular abundance profiles}

We solve the radiative transfer for $\mathrm{N}_{2} \mathrm{H}^{+}$and the $\mathrm{CO}$ isotopomers to derive their radial profiles of abundance. For this, we use a Monte Carlo code (Bernes 1979; Tafalla et al. 2002, 2004) and fit simultaneously both the radial profiles of integrated intensity and the emergent spectra of all transitions (the radial profiles have been derived with the same elliptical average as used for the continuum). Given the previous parameterization of the L1521E core, the only free parameters to fit the data are the abundances of each species.

To determine the $\mathrm{N}_{2} \mathrm{H}^{+}$abundance we use the 1-0 transition. This transition has 7 hyperfine components, and our model treats each component as a separate line, taking explicitly into account the hyperfine splitting of the first 3 rotational levels (see Tafalla et al. 2004 for further details). We 
have run this model assuming constant $\mathrm{N}_{2} \mathrm{H}^{+}$abundance, and as Fig. 3 shows, the predicted emission for a value of $2 \times 10^{-11}$ fits simultaneously the radial profile of integrated intensity and the central emerging spectrum. A constant $\mathrm{N}_{2} \mathrm{H}^{+}$abundance is also found in other cores, but the value we derive for L1521E is about 8 times lower than that derived for the L1498 and L1517B cores using the same code (Tafalla et al. 2004). This difference is highly significant and makes L1521E the starless core with the lowest $\mathrm{N}_{2} \mathrm{H}^{+}$abundance (note that the $\mathrm{N}_{2} \mathrm{H}^{+}$ abundances estimated by Tafalla et al. 2002 are artificially low because of their simplified treatment of the hyperfine structure). The low $\mathrm{N}_{2} \mathrm{H}^{+}$abundance explains the location of L1521E in the far left of Fig. 1.

To determine the abundance of the $\mathrm{CO}$ isotopomers, we now fit the radial profiles and emergent spectra of $\mathrm{C}^{18} \mathrm{O}(1-0)$, $\mathrm{C}^{18} \mathrm{O}(2-1), \mathrm{C}^{17} \mathrm{O}(1-0)$, and $\mathrm{C}^{17} \mathrm{O}(2-1)$. We first fix the $\mathrm{C}^{18} \mathrm{O} / \mathrm{C}^{17} \mathrm{O}$ abundance ratio to its ISM value (3.65, Penzias 1981), so we are left with one abundance profile as free parameter. In contrast with what is found in other cores, the L1521E data can be fit with a model of constant CO abundance. This is illustrated in Fig. 3 and makes L1521E the first core with no sign of $\mathrm{CO}$ depletion. Our best-fit abundance is $\mathrm{X}\left(\mathrm{C}^{18} \mathrm{O}\right)=0.7 \times 10^{-7}$, very similar to that found in the outer parts of L1498 and L1517B, although about 2.5 times lower than the standard determination by Frerking et al. (1982). This lower value may indicate a slight error in the dust parameters or that the Frerking et al. (1982) abundances need revision. In any case, it is the combination of high $\mathrm{CO}$ and low $\mathrm{N}_{2} \mathrm{H}^{+}$abundances what causes the hight $R$ ratio of L1521E.

Although an analysis of the CS emission in L1521E is out of the scope of this paper, we note that preliminary models indicate that this species also has constant abundance all the way to the core center (already suggested by the $\mathrm{C}^{34} \mathrm{~S}(2-1)$ emission in Fig. 2). CO and CS, therefore, seem not depleted in L1521E.

\section{An extremely young core}

The absence of CO (and CS) depletion in L1521E seems not to result from molecules finding it harder to freeze out in this core. Its central density is typical, as well as is its gas temperature of $10 \mathrm{~K}$ (the dust temperature cannot be much higher given the observed central density). The very low abundance of the late-time molecule $\mathrm{N}_{2} \mathrm{H}^{+}$further suggests that the lack of depletion in $\mathrm{L} 1521 \mathrm{E}$ results from this core being less chemically processed than others, as it has also been argued by Hirota et al. (2002) and Aikawa et al. (2003). L1521E, in fact, combines the lowest $\mathrm{C}^{18} \mathrm{O}$ depletion and lowest $\mathrm{N}_{2} \mathrm{H}^{+}$abundance, so it appears to be the less processed and therefore youngest core known.

To estimate how young L1521E is, we compare our observations with the predictions from the recent model of Aikawa et al. (2003), who have studied the evolution of a core undergoing Larson-Penston contraction taking into account both gas-phase and grain-surface chemical reactions. Although the exact form of contraction is unclear, this model seems preferable because it covers the chemical evolution of the gas since time prior to molecular depletion when it has an initial density of $10^{4} \mathrm{~cm}^{-3}$. Thus, we have taken the model abundance profiles at the earliest time calculated by the authors $\left(1.52 \times 10^{5} \mathrm{yr}\right.$, their Fig. 2a) and predicted intensities for the conditions of L1521E. In addition of having to multiply the predicted abundances by $\approx 3$ to approximately fit the data, we find that for $\mathrm{C}^{18} \mathrm{O}$ the slight central drop predicted by the chemical model is barely consistent with the data, and that the predicted central increase of $\mathrm{N}_{2} \mathrm{H}^{+}$abundance is already too pronounced (the central density at this early time is close to our estimate). This indicates that the model predicts more chemically processing at time $1.52 \times 10^{5} \mathrm{yr}$ than observed, even for the relatively fast LarsonPenston flow. With the caveat that the time estimate depends sensitively on the assumed sticking probabilities, we conclude that the comparison implies a contraction age of $\leq 1.5 \times 10^{5} \mathrm{yr}$.

Our contraction age for $\mathrm{L} 1521 \mathrm{E}$ seems consistent with the estimate by Lee \& Myers (1999) that the typical lifetime of starless cores is $0.3-1.6 \mathrm{Myr}$, as this core is younger than others. This age, however, seems incompatible with models of core formation via ambipolar diffusion, as they require evolutionary times of the order of $1 \mathrm{Myr}$ in the most favorable conditions (e.g., Ciolek \& Basu 2001). Thus, if L1521E has significantly contracted on a time scale as short as suggested by chemical models, a more dynamical picture seems needed. The collision of supersonic flows, as proposed by the supersonic turbulence scenario (e.g., Mac Low \& Klessen 2003), however, seems also inconsistent with the observations, given that the turbulent component of the linewidth $\left(\sigma \approx 0.11 \mathrm{~km} \mathrm{~s}^{-1}\right)$ is still subsonic, and that the velocity differences across the L1521E core are only of the order of the sound speed (a description of the core velocity structure will presented in a future paper). Clearly further analysis of L1521E is needed to understand its apparent rapid formation and lack of molecular depletion. At this point, it is suggestive to speculate that L1521E belongs to an up to now overlooked population of extremely young cores.

Acknowledgements. We thank the staffs of FCRAO and IRAM for help during the observations and Yuri Aikawa for advice on chemical models. M.T. acknowledges support from grant AYA2000-0927 of the Spanish DGES. The DSS was produced at the Space Telescope Science Institute under US Government grant NAG W-2166.

\section{References}

Aikawa, Y., Ohashi, N., \& Herbst, E. 2003, ApJ, 593, 906

André, P., Ward-Thompson, D., \& Motte, F. 1996, A\&A, 314, 625

Bergin, E. A., \& Langer, W. D. 1997, ApJ, 486, 316

Bernes, C. 1979, A\&A, 73, 67

Benson, P. J., \& Myers, P. C. 1989, ApJS, 71, 89

Caselli, P., Walmsley, C. M., Tafalla, M., Dore, L., \& Myers, P. C. 1999, ApJ, 523, L165

Ciolek, G. E., \& Basu, S. 2001, ApJ, 547, 272

Frerking, M. A., Langer, W. D., \& Wilson, R. W. 1982, ApJ, 262, 590

Hirota, T., Ito, T., \& Yamamoto, S. 2002, ApJ, 565, 359

Lee, C. W., \& Myers, P. C. 1999, ApJS, 123, 233

Mac Low, M.-M., \& Klessen, R. S. 2003, Rev. Mod. Phys., in press [astro-ph/0301093]

Penzias, A. A. 1981, ApJ, 249, 518

Tafalla, M., Myers, P. C., Caselli, P., Walmsley, C. M., \& Comito, C. 2002, ApJ, 569, 815

Tafalla, M., Myers, P. C., Caselli, P., \& Walmsley, C. M. 2004, A\&A, in press 